\title{
Influence of the grain size on the in vivo degradation behaviour of the magnesium alloy LAE442
}

Proc IMechE Part H:

$\mathrm{J}$ Engineering in Medicine

227(3) 317-326

(c) IMechE 2013

Reprints and permissions:

sagepub.co.uk/journalsPermissions.nav DOI: $10.1177 / 0954411912471495$

pih.sagepub.com

(SAGE

\author{
Berit Ullmann', Janin Reifenrath', Jan-Marten Seitz ${ }^{2}$, Dirk Bormann² and \\ Andrea Meyer-Lindenberg ${ }^{3}$
}

\begin{abstract}
The aim of this study was to investigate the differences in the in vivo degradation behaviour of magnesium implants with various grain sizes and damaged surfaces. For this purpose, three different LAE442 magnesium implants were produced: cast, single and double extruded implants, in order to obtain different grain sizes. Furthermore, defects were positioned on the surfaces of some of the single extruded implants. The initial stability was determined. Four pins of each implant material were implanted into rabbits' tibiae and regularly clinically, radiologically and $\mu$-computed tomographically investigated over a period of 27 weeks. Following explantation, investigations were carried out using stereo and scanning electron microscopy including energy-dispersive X-ray analyses. Weight and strength changes were measured. The double extruded implants possessing the finest grains exhibited the highest initial stability (I79.18 N). These implants demonstrated the lowest in vivo corrosion rates $(0.0134 \mathrm{~mm} /$ year $)$ and the least radiologically visible changes. The highest corrosion rate was computed for the implants possessing damaged surfaces. Radiologically discernible bone changes occurred at almost the same time as implant changes for all groups. Based on these results, the aim should be to produce fine-grained magnesium-based alloys for resorbable implants and to avoid any surface damage.
\end{abstract}

\author{
Keywords \\ Biodegradable, grain size, magnesium, in vivo
}

Date received: 4 September 2012; accepted: 26 November 2012

\section{Introduction}

Owing to its favourable properties such as good biocompatibility and mechanical stability, degradable implant materials based on magnesium are the focus of current biomedical materials research. ${ }^{1-8}$ Besides good biocompatibility, degradable implants must exhibit sufficient strength in order to be employed in load-carrying bone. Current degradable implant materials, such as ceramics and polymers, lack this strength. ${ }^{9,10}$ In contrast, magnesium alloys have similar mechanical properties to bone. ${ }^{2}$ By means of additionally alloying with other elements, such as calcium, lithium, aluminium or rare earths, the degradation properties and the strength of magnesium alloys can be influenced and thus lower the degradation rate and thereby reduce gas development. ${ }^{3,5,11-13}$ Besides the composition, surface processing and/or impairment of the surface, which could happen, for example, during the insertion of intramedullar osteosynthetic materials, also influence the magnesium implant's degradation. ${ }^{14,15}$ Roughened surfaces of $\mathrm{MgCa}$ cylinders lead to increased corrosion in vivo, ${ }^{14}$ while surface damage of AZ91 specimens showed a higher in vitro corrosion in simulated body fluid (SBF). ${ }^{15}$ The grain size as an additional parameter can also affect the degradation behaviour. The AZ31 magnesium alloy possessing finer grains demonstrated a better corrosion resistance in in vitro tests in SBF as the same alloy with coarser grains. ${ }^{16}$ Consequently, grain refinement could improve the corrosion behaviour and

\footnotetext{
'Small Animal Clinic, University of Veterinary Medicine Hannover, Hannover, Germany

${ }^{2}$ Institute of Materials Science, Hannover Centre for Production Technology, Leibniz Universität Hannover, Garbsen, Germany ${ }^{3}$ Clinic for Small Animal Surgery and Reproduction, Centre of Clinical Veterinary Medicine, Faculty of Veterinary Medicine, LudwigMaximilians-Universität München, Munich, Germany
}

\section{Corresponding author:}

Janin Reifenrath, Small Animal Clinic, University of Veterinary Medicine Hannover, SFB 599, Bünteweg 9, 30559 Hannover, Germany.

Email: janin.reifenrath@tiho-hannover.de 
possibly help to systematically control the corrosion. Grain refinement can, among others, be carried out by means of extrusion, ${ }^{17,18}$ which leads to recrystallization and the formation of new and fine grains via mechanical and thermal processes. ${ }^{18,19}$

The promising magnesium-based alloy LAE442 (containing $4 \mathrm{wt} \%$ lithium, $4 \mathrm{wt} \%$ aluminium and $2 \mathrm{wt} \%$ rare earths) demonstrated slow degradation with a subsequent low impact on the surrounding tissue that would allow for an adequate bone healing, as well as good biocompatibility. ${ }^{1,20}$ The influence of various grain sizes on this alloy's degradation and biocompatibility has not been previously tested. Therefore, it was the objective of this study to investigate the degradation behaviour of LAE442 with various grain sizes over a period of 6 months in vivo. Additionally, the influence of implant surface damage on the degradation rate was tested.

\section{Material and methods}

\section{Implant material}

All implants used in this study were made of the magnesium-based alloy LAE442 (4 wt \% lithium, $4 \mathrm{wt} \%$ aluminium and $2 \mathrm{wt} \%$ rare earths. ${ }^{21} \mathrm{~A}$ total of 42 implants were manufactured using the casting process in a protective gas atmosphere of atal. Further processing prior to turning the material to the final implant's size (25 $\mathrm{mm}$ in length and $2.5 \mathrm{~mm}$ in diameter) varied, such that four different implant types were generated:

type 1: no extrusion;

type 2: extrusion from 120 to $30 \mathrm{~mm}$ at $350{ }^{\circ} \mathrm{C}$;

type 3 : first extrusion from 120 to $30 \mathrm{~mm}$ at $350^{\circ} \mathrm{C}$, second extrusion from 30 to $10 \mathrm{~mm}$ at $350{ }^{\circ} \mathrm{C}$;

type 4: extrusion from 120 to $9 \mathrm{~mm}$ at $350^{\circ} \mathrm{C}$; after obtaining the implant's final size, four longitudinal notches at the proximal end and three transverse notches in the middle of the implant were produced using a 22-gauge needle (Braun, Germany).

All implants were weighed, washed with acetone and distilled water, packed in special sterilization bags and sterilized using gamma radiation (27.9 kGy; BBF Sterilisationsservice, Germany). Using a universal test machine (Zwick, Germany), the mechanical properties of four implants of type 1 , type 2 and type 3 were determined by four-point bending tests as previously described. ${ }^{1,3}$ Therefore, the implants were placed centrally on the abutments with a distance of $15 \mathrm{~mm}$ between supports. A displacement transducer measured the distance during the test cycle. Before the test, a preload of $2.5 \mathrm{~N}$ was applied. The subsequent downward movement of the forming punch was at a constant speed of $1 \mathrm{~mm} / \mathrm{min}$. Termination criteria were a sudden drop of force of $10 \%$ or a bending of $5 \mathrm{~mm}$. The maximum force $(F \max (\mathrm{N}))$ and the bending displacement at fracture (å-Fmax (mm)) were recorded.
In metallographic examinations, the grain size of type 1, type 2 and type 3 implants was determined three times according to DIN EN ISO 643, generally at a magnification of 100:1. The number of grains $\left(\mathrm{K}_{\mathrm{W}}\right)$ within a gauge circle of $5000 \mathrm{~mm}^{2}$ (surface $\mathrm{A}_{\text {circle }}$ ) corresponding to a diameter of $79.8 \mathrm{~mm}$ was counted, while the grains cut by the circle's edge $\left(K_{c}\right)$ were added to the total number as only half grains. The computation of the mean grain size $\left(\mathrm{K}_{\text {mid }}\right)$ was performed subject to equation (1) and valid according to DIN EN ISO 643

$$
\mathrm{K}_{\text {mid }}=\sqrt{\frac{\mathrm{A}_{\text {Circle }}}{\mathrm{K}_{\mathrm{W}}+\frac{\mathrm{K}_{\mathrm{C}}}{2}}}
$$

The surface of every implant was examined microscopically (Axio CamMRC; Carl Zeiss AG, Germany) using 5- to 40-fold magnifications. The implants' surfaces were evaluated descriptively.

\section{Animal model}

The animal tests were approved by the local government of Hannover (reference number 33.9-42502-04-07/ 1363). Four cylinders of each implant type were inserted longitudinally in the medullary cavity of adult New Zealand white rabbits (Charles River, Deutschland). Each animal received two implants of the same type. Surgery was carried out as previously described. ${ }^{1}$ The implantation period was 6 months. Clinical examinations during the post-operative period focussed on redness, soft/hard swellings, lameness, development of gas, purulence or suture dehiscence. Both tibiae were radiologically examined weekly in the mediolateral and anteroposterior beam path using $48 \mathrm{kV}$ and $6.3 \mathrm{mAs}$ (Practix 160; Philips, Germany). The implants were assessed using semi-quantitative scores described by Huehnerschulte et al. ${ }^{3}$ Three different parts were assessed: the implant itself (structural changes), the bone changes of the diaphysis, the corticalis and at the implantation site and gas formation. Score values were given from 0 (no change) to 3 (severe change). ${ }^{3}$ All parameters were summed to a cumulative score and depicted graphically.

According to Huehnerschulte et al., ${ }^{3}$ every second week, up to week 12, and then every fourth week as well as on the day of euthanization, $\mu$-computed tomographies ( $\mu \mathrm{CT}$ s) were performed (XtremeCT; Scanco Medical, Switzerland) under general anaesthesia. The implants were manually contoured and subsequently measured by means of the $\mu \mathrm{CT}$ software evaluation program V6.1 (XtremeCT; Scanco Medical). The threshold was adapted for each group (type 1, type 2: 138; type 3: 236), and the following parameters were evaluated: volume, density and true three-dimensional (3D) thickness as well as the standard deviation (SD) of the true 3D thickness. The latter equates to the variance of the maximum diameters within an implant ${ }^{3}$ and will subsequently be named 'variance'. It displays the homogeneity of the overall degradation of an 
implant. ${ }^{3,22}$ The implant's corrosion rate was calculated using the in vivo $\mu \mathrm{CT}$ data according to the following formula: $\mathrm{CR}=\Delta \mathrm{V} /(\mathrm{A} \times \mathrm{t}){ }^{23}$ Here, $\mathrm{CR}(\mathrm{mm} /$ year $)$ is the corrosion rate, $\Delta \mathrm{V}\left(\mathrm{mm}^{3}\right)$ is the volume loss, A $\left(\mathrm{mm}^{2}\right)$ is the area that was exposed to the corrosion and $t$ (days) is the implantation period.

\section{Evaluation of the implants ex vivo}

After 6 months, the animals were anaesthetized using sketamine hydrochloride $(20 \mathrm{mg} / \mathrm{kg}$; CP-Pharma, Germany) and xylazinhydrochloride $(5 \mathrm{mg} / \mathrm{kg}$; Serumwerk Bernburg AG, Germany) and painlessly euthanized by an intracardial injection of pentobarbital (230 mg/kg; Narkodorm ${ }^{\circledR}$ CP-Pharma, Germany). The tibiae were opened longitudinally using a Dremel rotary cutter, and the implants were carefully removed and examined microscopically.

For further evaluation of the implant's surface, one implant of each group was examined by means of scanning electron microscopy (SEM) (LEO VP 1455; Zeiss, Germany). At selected locations, an element analysis was performed using energy-dispersive X-ray (EDX) spectroscopy (X-flash Detector 5010; Bruker, Germany). SEM images with Rutherford Backscattering Spectroscopy (RBS) and Variable Pressure Secondary Electrons (VPSE) were taken (resolution: $5 \mathrm{~nm}$ ). The evaluation was performed descriptively.

To evaluate the volume loss of the implants, the organic material was washed off with hydrofluoric acid $(40 \%))^{3,24}$ Following this, the implants were once more microscopically and scanning electron microscopically examined and weighed (AB204-S/Fact; Metler Toledo, Germany). The average weight loss of each group is expressed as the difference in percent of the remaining implant, after hydrofluoric acid treatment, to the initial weight before implantation. Subsequently, the mechanical properties were determined analogous to the mechanical testing in the initial condition.

\section{Statistics}

Statistical analysis was performed with Microsoft Office Excel ${ }^{\circledR}$ Version 2003 and SPSS ${ }^{\circledR}$ Version 17.0. After testing for normal distribution, statistical differences for weight changes, volume calculation and true $3 \mathrm{D}$ thickness, including variances, were determined by means of t-tests for paired samples. Data for mechanical properties were analysed using t-test for independent samples. The evaluation of density changes within a group was performed with tests according to Wilcoxon. Comparisons between the groups were statistically investigated by means of a univariate analysis of variance (ANOVA) with subsequent post hoc tests (Tukey, Games-Howell). Values of $p \leqslant 0.05$ and $p \leqslant 0.01$ were interpreted as significant and highly significant, respectively.

\section{Results}

\section{Examination of the initial implants}

Three-point bending. Type 1 implants with $126.01 \mathrm{~N}$ (SD 12.87) exhibited a significantly lower maximum force (Fmax) than type 2 (168.11 N (SD 4.79)) and type 3 (179.18 N (SD 9.83)) implants. Hence, on average, type 3 implants possessed a higher Fmax than type 2 implants, but no significance could be evaluated.

The average bending displacement at fracture (å-Fmax (mm)) was $0.5 \mathrm{~mm}$ (SD 0.036) for type 3 implants and was significantly lower $(p=0.016$ to $p<0.001)$ than those for type $2(1.85 \mathrm{~mm}$ (SD 0.43)) and type 1 (1.31 mm (SD 0.42)) implants. No significance could be calculated between type 1 and type 2 implants.

Metallographic examination. The average grain size differed between the groups with a high significance ( $\mathrm{p}<0.01)$. Type 1 implants possessed the largest average grain size $(36.85 \mu \mathrm{m}$ (SD 2.86)) (Figure 1(a)) in comparison to type $2(25.32 \mu \mathrm{m}$ (SD 2.67)) (Figure 1(b)) and type 3 implants (11.27 $\mu \mathrm{m}$ (SD 0.6)) (Figure 1(c)).

\section{Examination of the explanted pins Microscopic examination. Prior} to implantation, a distinct difference between the implant types could be seen. Type 1 implants exhibited the broadest transverse grooves (dependent on manufacturing) as well as more unevenness around and on these grooves (Figure 2(a)). Type 3 implants possessed the narrowest transverse grooves and the most homogeneous structure (Figure 2(c)). The notches on the surface of type 4 implants could be clearly identified (Figure 2(d)).

\section{Animal model}

Clinical examination. All animals showed minor-tomoderate redness and swelling on both legs, which disappeared no later than the 27th day after surgery. Minor hard but painless swellings appeared around the implantation site, on average, on the fifth day. Moderate hard but painless swellings could be observed between the 9 th and the 26th day and were still present in six animals on the day of euthanasia. One animal, with type 1 implants, showed lameness, which altered from minor to severe, from day 31 to day 68 . Depending on its severity, the lameness was treated with meloxicam $\left(0.15 \mathrm{mg} / \mathrm{kg}\right.$, Metacam ${ }^{\circledR}$; Boehringer Ingelheim, Ingelheim, Germany) and buprenorphine hydrochloride $(0.05 \mathrm{mg} / \mathrm{kg}$; Bayer AG, Leverkusen, Germany). The development of gas in the form of subcutaneous emphysema could not be observed in any of the animals.

Radiologic examination. The earliest radiological changes in the form of growths at the diaphysis could be seen in week 4 (type 2 implants). Structural changes of the 


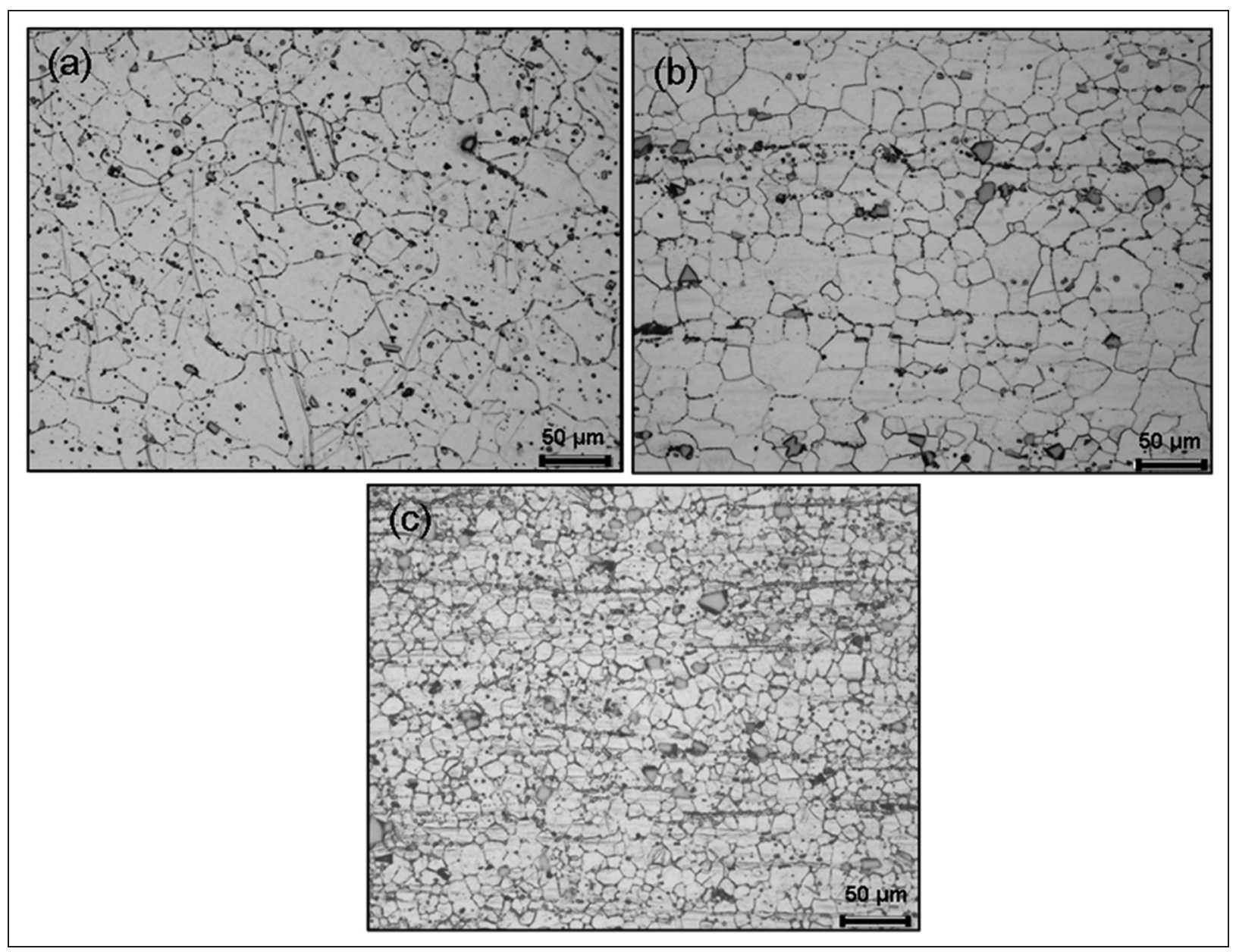

Figure I. Metallographic images with different grain sizes: (a) type I, (b) type 2 and (c) type 3.

implants, appearing as brightening, were first seen (week 8) most distinctly in implants of type 1. The structure of type 3 implants remained constant for the longest period of time (until week 17). On average, minor, diffuse intramedullary gas development could be seen intermittently in all groups from the eighth week up until the end of the observation period. Minor, inhomogeneous structural changes of the corticalis as well as growth at the implantation site played a minor role in the total score, which increased with small fluctuations in all groups over the time period (Figure 3). On average, implants of type 3 showed the lowest total score values, as well as the slightest bone changes, over the entire observation period (Figure 3). Looking at the implant changes and the bone changes separately, it could be seen that they occurred at similar points in time.

In vivo $\mu C T$. The evaluation of the in vivo $\mu \mathrm{CT}$ showed the lowest initial density for implants of type 4, whereas highest density for implants of type 3 (Table 1). Over time, a minor loss of density but with no significance could be determined. As for the volume, a significant loss could be calculated for every implant type. The volume loss was most distinct for type 4 implants in contrast to type 3 implants, which had the lowest volume loss (Table 1). Over time, the diameter (true 3D thickness) decreased, mainly homogeneously, in all implant types. Here again, the type 4 implants showed the highest and type 3 the lowest loss. The variance of the diameter increased predominantly continuously in all implant types over time. Throughout the examination, type 3 implants had the lowest variance values (Table 1).

The calculated corrosion rates, based on the $\mu \mathrm{CT}$ data (Table 1), showed a decrease over time. The highest corrosion rate after 2 weeks was determined for implants with defects (type 4; $0.121 \mathrm{~mm} /$ year). On average, type 3 implants exhibited the lowest corrosion rate, which was $0.0134 \mathrm{~mm} /$ year, at the end of the observation period. The corrosion rate of type 1 and type 2 implants was 0.035 and $0.025 \mathrm{~mm} /$ year, respectively, at the end of the observation period, whereas the implants with defects again had the highest corrosion rate with $0.04 \mathrm{~mm} /$ year.

\section{Examination of the explanted pins}

Microscopic examination. Directly after explantation, irregular, uneven brown and white deposits as well as cracks could be microscopically observed on the 


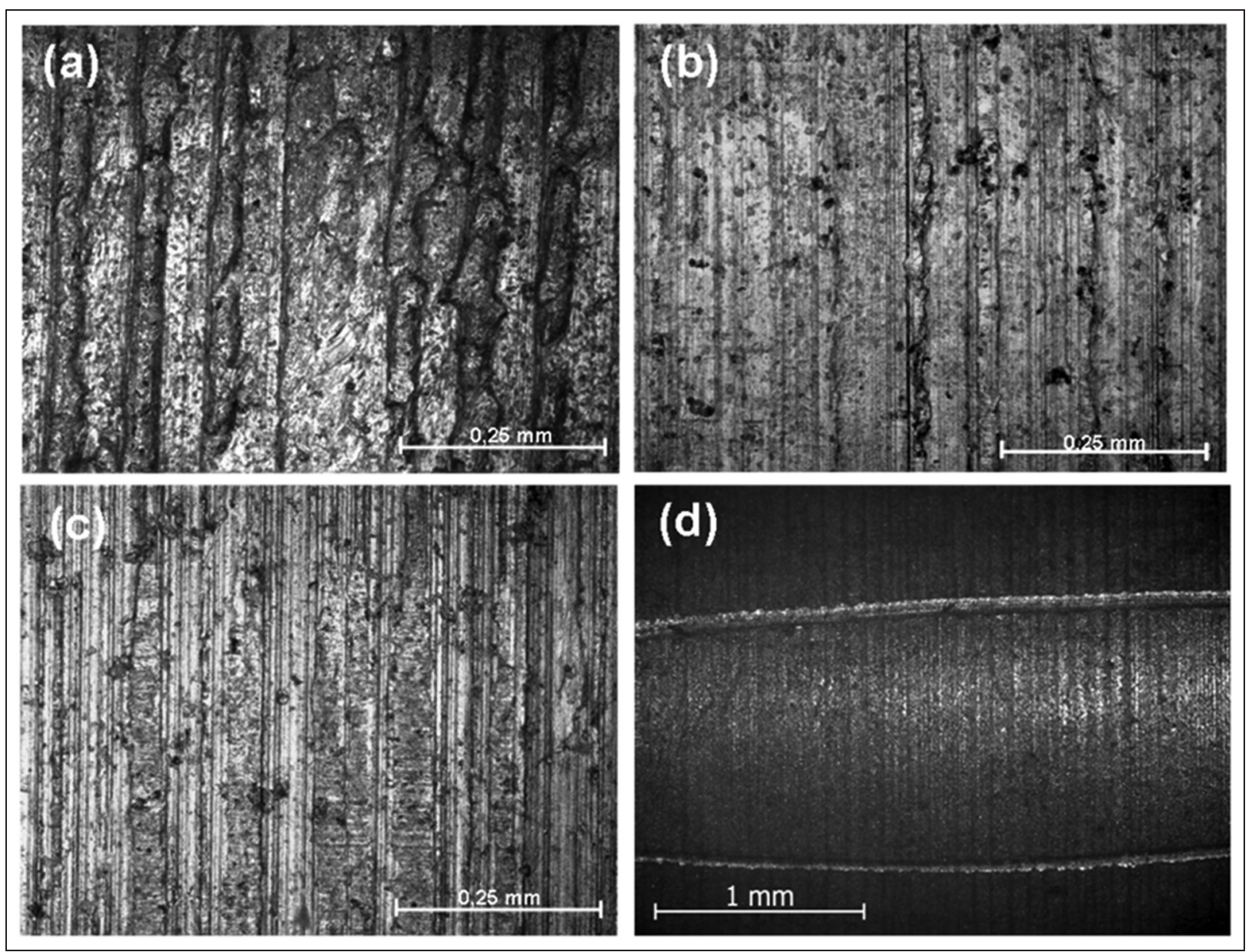

Figure 2. Microscopic images of the implants' surfaces prior to implantation: (a) type I ( $\times 20$ magnification), (b) type 2 ( $\times 20$ magnification), (c) type 3 ( $\times 20$ magnification) and (d) type 4: artificial defects (longitudinal notches) at the end of the implants $(\times 5$ magnification $)$.

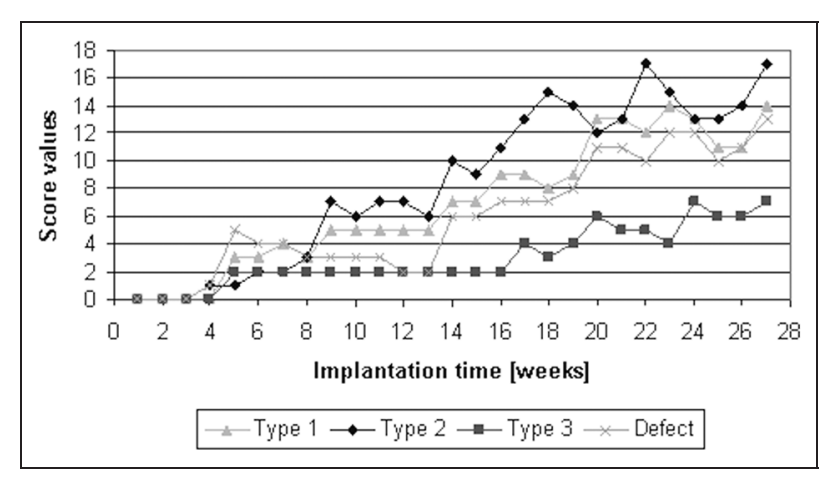

Figure 3. Total radiological scores for the different groups over time; a low total score represents only little changes of the implant and the bone.

implants. Moreover, type 3 implants showed fine transverse grooves. The defects, appearing as longitudinal notches, of the type 4 implants could still be seen on three implants. The transverse notches could no longer be seen on any of the implants.

After treating with hydrofluoric acid, an uneven surface (type 1 and type 4), rounded ends (type 1 and type 2 ), longitudinal grooves (type 2, type 3 and type 4 ) as well as pitting corrosion (in every implant type) could be detected. One half of one type 3 implant showed an inhomogeneous corrosion and a tapered end. The other half of the same implant showed none of these changes. In addition to this, this morphology could not be seen prior to the hydrofluoric acid treatment. All other implants exhibited none of these distinct differences.

SEM examination. Prior to treating with hydrofluoric acid, all implants examined in the SEM had an uneven and clump-like surface. The implants' cylindrical form was almost retained. It was possible to detect regions containing rare earths as well as calcium- and phosphate-rich deposits on the implants' surface by means of EDX analyses.

After treating with hydrofluoric acid, the type 1 implant showed an uneven surface, pitting corrosion, oblique transverse grooves as well as distinct differences between the implants' ends (Figure 4(a)). The implants of the other types showed distinct longitudinal lines, clump-like structures and also pitting corrosion (Figure 4(b)). The proximal end of the type 3 implant had longitudinal grooves and deposits, and the distal end exhibited clump-like structures (Figure 4(c)). The 

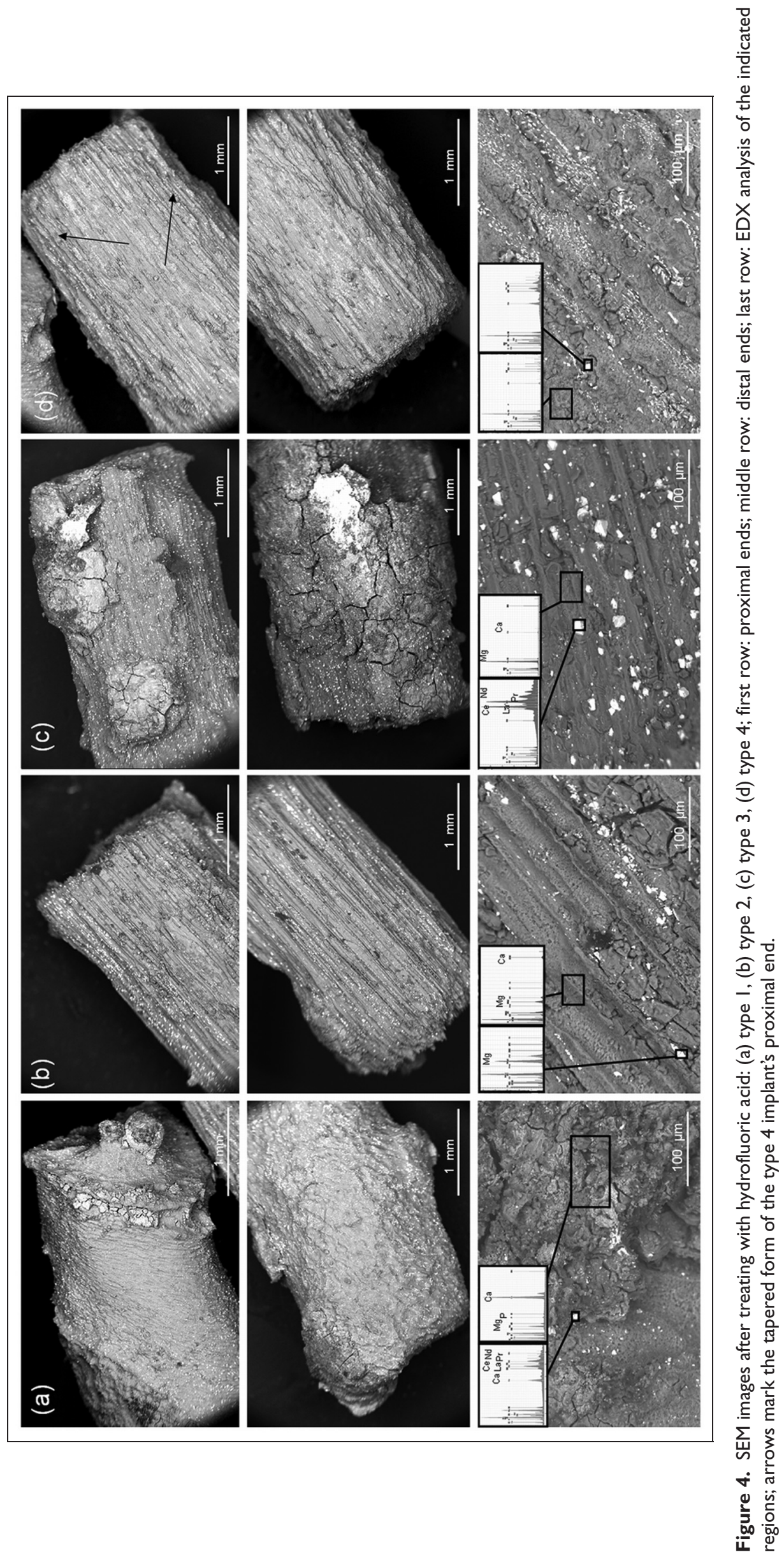
Table I. $\mu$-computed tomographic evaluation of density, volume, true 3D thickness and its variance.

\begin{tabular}{|c|c|c|c|c|}
\hline Parameter & Implant & Value at implantation & Value at explantation & Difference in \% \\
\hline \multirow[t]{4}{*}{ Density $\left(\mathrm{mgHA} / \mathrm{cm}^{3}\right)$} & Type I & $1202.46 \pm 33.83^{\mathrm{a}}$ & || $57.33 \pm\left. 38.8\right|^{\mathbf{A}}$ & -3.75 \\
\hline & Type 2 & $1311.0 \pm 135.65^{b c}$ & $1260.07 \pm 126.72^{B C}$ & -3.89 \\
\hline & Type 3 & $2289.39 \pm 51.46^{\text {abd }}$ & $2254.14 \pm 65.72^{\mathrm{ABD}}$ & -1.54 \\
\hline & Type 4 & $1 / 23.08 \pm 12.05^{\mathrm{cd}}$ & $1104.1 \pm 16.48^{C D}$ & -1.69 \\
\hline \multirow[t]{4}{*}{ Volume $\left(\mathrm{mm}^{2}\right)$} & Type I & $128.26 \pm 5.64^{*}$ & $124.54 \pm 3.76^{*}$ & -2.91 \\
\hline & Type 2 & $129.85 \pm 3.28 *$ & $127.24 \pm 3.69 *$ & -2.01 \\
\hline & Type 3 & $133.30 \pm 3.78 *$ & $|3| .89 \pm 4.3 *$ & -1.07 \\
\hline & Type 4 & $130.95 \pm 0.8^{*}$ & $126.7 \mid \pm 1.7 *$ & -3.24 \\
\hline \multirow{4}{*}{ True 3D thickness (mm) } & Type I & $2.3434 \pm 0.08^{a}$ & $2.2828 \pm 0.02^{A}$ & -2.59 \\
\hline & Type 2 & $2.3822 \pm 0.01 *$ & $2.3 \mid 43 \pm 0.03 * B$ & -2.85 \\
\hline & Type 3 & $2.4730 \pm 0.05^{* a}$ & $2.4315 \pm 0.06 * A B C$ & -1.68 \\
\hline & Type 4 & $2.4116 \pm 0.02$ & $2.3100 \pm 0.08^{C}$ & -4.23 \\
\hline \multirow{4}{*}{ Variance of true 3D thickness } & Type I & $0.1482 \pm 0.06 *$ & $0.2830 \pm 0.08 *$ & +91 \\
\hline & Type 2 & $0.2047 \pm 0.05$ & $0.2500 \pm 0.03$ & +22 \\
\hline & Type 3 & $0.1291 \pm 0.06$ & $0.2009 \pm 0.09$ & +56 \\
\hline & Type 4 & $0.1713 \pm 0.02$ & $0.2790 \pm 0.09$ & +63 \\
\hline
\end{tabular}

Small letters: significance between the groups at implantation; capital letters: significance between the groups at explantation; plain text: significant $(p \leqslant 0.05)$; bold: highly significant $(p \leqslant 0.01)$; 3D: three-dimensional.

*Significance between value at implantation and explantation.

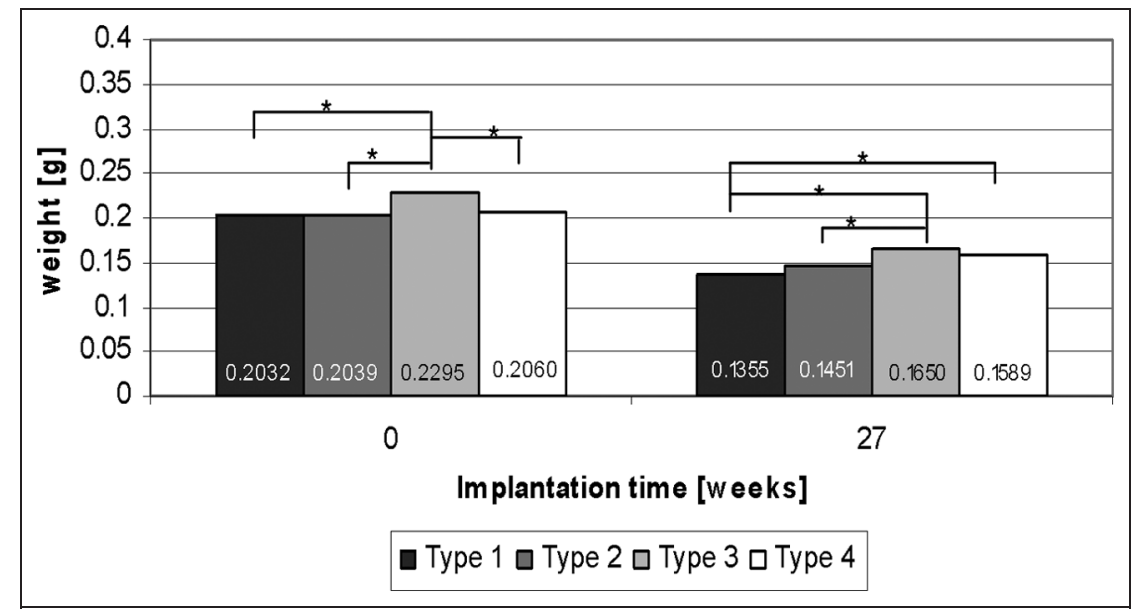

Figure 5. Weight determination of the different implant types, before and after trial; significances $(p \leqslant 0.05)$ are marked with asterisk.

proximal end of the type 4 implant had a distinctly tapered form.

Weight determination. A significant $(p=0.003$ to $p<0.001$ ) weight loss could be detected in all implants after explantation (Figure 5). Type 1 implants had an average loss of $33.23 \%$ and thereby the highest weight loss. Implants of type 2, type 3 and type 4 showed mean weight losses of $28.20 \%, 28.1 \%$ and $22.85 \%$, respectively.

Three-point bending test. The maximum force after explantation and treating with hydrofluoric acid was significantly $(p=0.001$ to $p<0.001)$ lower than the initial state for all implants (Figure 6). Type $1(77.31 \mathrm{~N}$ (SD 18.93)) implants had significantly lower values after explantation than type $2(99.38 \mathrm{~N}$ (SD 6.85), $p=0.031)$ and type 3 (105.31 N (SD 9.39), $p=0.014)$ implants. No significance could be determined between

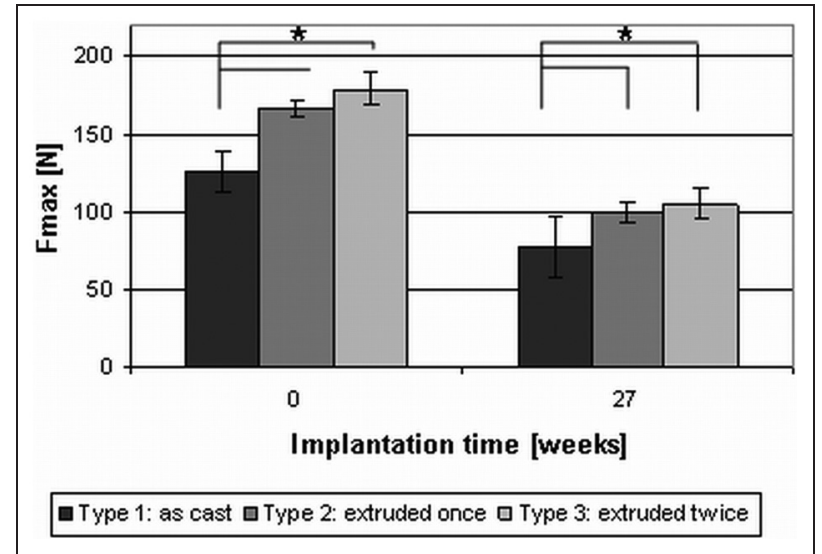

Figure 6. Fmax: initial state and at the end of the observation period; significances $(p \leqslant 0.05)$ are marked with asterisk.

types 2 and 3 implants, but, on average, type 3 implants had higher values than type 2 implants (Figure 6). 
The deflection at rupture after the observation period had increased for all implant types. However, a significant change $(1.72 \mathrm{~mm}$ (SD 0.41), $p=0.01)$ in comparison to the initial value $(0.5 \mathrm{~mm}$ (SD 0.42)) could only be detected for type 3 implants. No significance was detected between the different implant types at the end of the observation period.

\section{Discussion}

The objective of this study was to investigate the influence of various grain sizes and that of surface damage on the in vivo degradation behaviour of the magnesium alloy LAE442. Within this scope, the grain sizes, mechanical properties and changes in volume, density and weight were measured, and radiological investigations were performed. The implants' morphology was established by means of SEM and microscopy images.

In comparing the grain sizes in this study, the coarsegrained implants showed the most severe loss of weight and volume as well as the highest corrosion rate and therefore a rapid degradation, while the fine-grained (type 3) implants exhibited the lowest corrosion rates and the least loss of volume and weight. In in vitro studies, Alvarez-Lopez et al. ${ }^{16}$ also found a better corrosion resistance for the fine-grained AZ31 alloys. The authors attributed this to the strong negative corrosion potential and to the associated rapid electrochemical reaction with the formation of a protective layer from the corrosion products. ${ }^{16}$ The results of the present study show that such an effect of the grain size on the corrosion can also be confirmed in vivo.

It initially appears to be contradictory that a finegrained implant was more strongly degraded on one side. Following explantation and treating with hydrofluoric acid, a partly very inhomogeneous, uneven surface was visible. In contrast, prior to treating with hydrofluoric acid, there was no evidence for a nonuniform degradation of this type 3 implant. Quite the opposite; the in vivo variance of the maximum diameter was always lower than those of the other groups. The volume, density and weight loss were also lower in comparison with the coarse-grained implants. Moreover, fine-grained implants exhibited the lowest corrosion rates. Subsequent to treating with hydrofluoric acid, the other implants of types 1, 2, 3 and 4 exhibited a more clearly visible corrosion than prior to the hydrofluoric acid treatment as well. As previously mentioned, it is described in the literature that grain refining leads to an elevated in vitro corrosion resistance and, moreover, to a more uniform degradation. ${ }^{8,16,25}$ A possible cause for the inhomogeneous degradation of one type 3 implant, which is visible following hydrofluoric acid treatment, can be sought in the formation of phases in a magnesium alloy. Magnesium-aluminium alloys mainly consist of an $\alpha$-matrix, â-phases and other intermetallic particles on which corrosion pits frequently develop. ${ }^{5}$ For small grain sizes, the â-phase forms an almost continuous network and can lead to certain corrosion protection. ${ }^{26}$ Moreover, the corrosion occurs, above all, in and from $\alpha$-grain to $\alpha$-grain and frequently stops at the grain boundaries before they reach the $\beta$-phase. ${ }^{25}$ Consequently, a brittle, honeycombshaped structure develops in vitro by means of the remaining â-phase. ${ }^{25}$ Prior to treating with hydrofluoric acid, the corrosion products, bonded with organic material, could be held in situ by means of the â-phase and were only then dissolved away by the hydrofluoric acid treatment. This would explain why no deep corrosion could be discerned on the implant prior to treating with hydrofluoric acid.

The computed in vivo corrosion rate demonstrated the highest values for implants possessing defects (type 4). In the course of time, the corrosion rate of all the investigated implants decreased. This was also described by other authors for magnesium alloys and is attributed by Witte et al. (for the alloys LAE442 and AZ91D) to the formation of a stable corrosion layer subsequent to the initial corrosion directly following surgery..$^{20,21,23}$ Furthermore, the formation of a calcium phosphate film on the magnesium implants' surface impedes the in vitro formation of local corrosion. ${ }^{15}$ Witte et al. ${ }^{4}$ assume that this effect could also occur in vivo. In this study, it was possible to verify the presence of calcium and phosphorus on all implants using the SEM/EDX analyses. Other in vivo studies also substantiate the verification of calcium and phosphorus on the surfaces of implants based on magnesium in guinea pigs (6 and 8 weeks) ${ }^{4}$ and rats $(6 \text { months })^{27}$ as well as in vitro in SBF. ${ }^{28,29}$ However, the propagation of preexisting notches and cracks is more rapid in vitro than the formation of the protective calcium phosphate film. ${ }^{15}$ This effect could also occur in vivo and explain the highest average corrosion rates of defective implants (type 4) found in this study. The significant tapering and thereby marked degradation of the proximal end of the type 4 implants can also be explained by means of this.

The corrosion rate calculated on the basis of $\mu \mathrm{CT}$ data regarding the volume loss differed obviously from the corrosion measured by the weight loss method. Although the weight loss method is commonly used to reliably quantify the implant degradation of either in vitro or in vivo studies, ${ }^{25,30}$ it has to be considered that the removal of the adherent corrosion products prior to weighing could possibly damage the implant or does not succeed completely. With the used in vivo $\mu \mathrm{CT}$, no previous treatment is necessary to evaluate the implants, but the resolution is not sufficient to distinguish between the implant itself and the corrosion layer. Huehnerschulte et al. ${ }^{3}$ reported on similar results, which could explain the differences between the results of both evaluation methods. In addition, the SEM analysis supports this, which showed distinct differences of the implants surfaces prior to and after hydrofluoric acid treatment. Other studies calculated the corrosion rate of magnesium alloys after in vivo 
implantation and gained different results depending on the method used. ${ }^{3,23,31}$ In vivo $\mu \mathrm{CT}$ data-based corrosion rates were generally slower ${ }^{3}$ than corrosion rates calculated via weight loss method $^{31}$ or volume loss/pitting factor. ${ }^{23}$ All studies including the present examinations show in common that the corrosion rate changes over the course of time, and therefore, the definite end point of degradation has to be determined by animal studies that last long enough to show the total implant degradation.

Radiologically identifiable changes in the implant and the bone found in this study were also described by other authors for in vivo intramedullar implants that are based on magnesium. 3,4,6,7 Diaphyseal changes occurred in the current study almost synchronously with the implant's changes. This could be explained by means of the gas that arises during the corrosion of the magnesium ${ }^{2,4,32,33}$ and that is diffused and resorbed at various rates depending on the surrounding tissue. ${ }^{4,8}$ In this study, it was possible to radiologically see small amounts of gas in the bone's medullary cavity from, on average, the eighth week. Huehnerschulte et al. ${ }^{3}$ also found gas by means of $\mu \mathrm{CT}$ in bone in in vivo tests with magnesium-based alloys in rabbits' tibiae but not in the surrounding tissue. Increased pressure could be exerted on the bone due to the gas accumulated in the medullary cavity. The bone reacts to mechanical loading by increasing the bone tissue. ${ }^{34,35}$ This could explain the chronological correlation of the additional diaphyseal formation with the implants' degradation. Another possible explanation could be the elevated activity of osteoblasts, which was assumed by Witte et al., ${ }^{4,36}$ due to the magnesium ions. In an in vivo study with guinea pigs' femurs, it was possible for Witte et al. ${ }^{4}$ to observe the additional formation of endosteal and periosteal bone after 6 and 8 weeks.

Regarding the mechanical properties in this study, the double extruded implants (type 3, finest grain) exhibited, as expected, the highest maximum force (Fmax) in their initial state, which is an advantage for the stable care of fractures. It is described several times in the literature that grain refinement is induced by means of plastic deformation that occurs during extru$\operatorname{sion}^{18,19}$ by means of which the mechanical properties are improved. ${ }^{5,18,19,37}$ The elongation at rupture, and therefore the implants' ductility, was the lowest for implants possessing the finest grains. This is to be favourably evaluated for osteosynthesis since the implant's deformation would be disadvantageous for stable care of the fracture. Following the testing, all the implants exhibited higher elongation values in comparison to their initial states. These results, for the type 3 implants, were even statistically significant. Krause et al. ${ }^{21}$ also found slightly higher ductility values following the implantation of WE43 implants after an implantation period of 6 months compared to those after 3 months. This can be explained using the reduced diameter that is induced by the degradation process and permits a larger deformation during bending. ${ }^{21}$ Why specifically those implants possessing the finest grains exhibited higher elongations to rupture than in their initial state cannot be explained with the aid of the current work and remains the reserve for further investigations.

\section{Conclusion}

The in vitro results for the corrosion properties of alloys possessing various grain sizes and defects are also reflected in the in vivo results. Implants having finer grains showed, on average, a slower corrosion rate and a better clinical tolerance than implants possessing coarse grains. Defects on the implant's surface lead to an initially accelerated corrosion in comparison to implants without defects. Therefore, attention should be focussed on a fine-grained alloy with an intact, smooth surface for applications as a medical product.

\section{Funding}

All work was carried out within the collaborative research centre 599, which is sponsored by the German Research Foundation (DFG).

\section{Acknowledgements}

The authors would like to thank Svenja Pfarr, Harald Kernbach, Jutta Baden, Anja Krabbenhöft and Roger Donoghue for their excellent technical support.

\section{References}

1. Thomann M, Krause C, Bormann D, et al. Comparison of the resorbable magnesium alloys LAE442 und $\mathrm{MgCa} 0.8$ concerning their mechanical properties, their progress of degradation and the bone-implant-contact after 12 months implantation duration in a rabbit model. Mater Sci Eng Technol 2009; 40: 82-87.

2. Staiger MP, Pietak AM, Huadmai J, et al. Magnesium and its alloys as orthopedic biomaterials: a review. Biomaterials 2006; 27: 1728-1734.

3. Huehnerschulte TA, Angrisani N, Rittershaus D, et al. In vivo corrosion of two novel magnesium alloys ZEK100 and AX30 and their mechanical suitability as biodegradable implants. Materials 2011; 4: 1144-1167.

4. Witte F, Kaese V, Haferkamp H, et al. In vivo corrosion of four magnesium alloys and the associated bone response. Biomaterials 2005; 26: 3557-3563.

5. Zeng R, Dietzel W, Witte F, et al. Progress and challenge for magnesium alloys as biomaterials. Adv Eng Mater 2008; 10: B3-B14.

6. Reifenrath J, Krause A, Bormann D, et al. Profound differences in the in-vivo-degradation and biocompatibility of two very similar rare-earth containing $\mathrm{Mg}$-alloys in a rabbit model. Materwiss Werksttech 2010; 41: 1054-1061.

7. Hampp C, Ullmann B, Reifenrath J, et al. Research on the biocompatibility of the new magnesium alloy LANd442 - an in vivo study in the rabbit tibia over 26 weeks. Adv Eng Mater 2012; 14: B28-B37.

8. Witte F, Hort N, Vogt C, et al. Degradable biomaterials based on magnesium corrosion. Curr Opin Solid State Mater Sci 2008; 12: 63-72. 
9. Salgado AJ, Coutinho OP and Reis RL. Bone tissue engineering: state of the art and future trends. Macromol Biosci 2004; 4: 743-765.

10. Hofmann GO. Biodegradable implants in traumatology: a review on the state-of-the-art. Arch Orthop Trauma Surg 1995; 114: 123-132.

11. Song $\mathrm{G}$ and Atrens A. Understanding magnesium corrosion - a framework for improved alloy performance. $A d v$ Eng Mater 2003; 5: 837-858.

12. Yazhong Z, Pan F, Peng J, et al. Effect of neodymium on the as-extruded ZK20 magnesium alloy. $J$ Rare Earth 2010; 28: 631-635.

13. Tong G, Liu H and Liu Y. Effect of rare earth additions on microstructure and mechanical properties of AZ91 magnesium alloys. T Nonferr Metal Soc 2010; 20: 336340 .

14. Von der Höh N, Bormann D, Lucas A, et al. Influence of different surface machining treatments of magnesiumbased resorbable implants on the degradation behavior in rabbits. Adv Eng Mater 2009; 11: B47-B54.

15. Kannan MB, Singh Raman RK, Witte F, et al. Influence of circumferential notch and fatigue crack on the mechanical integrity of biodegradable magnesium-based alloy in simulated body fluid. J Biomed Mater Res B Appl Biomater 2010; 96: 303-309.

16. Alvarez-Lopez M, Pereda MD, Del Valle JA, et al. Corrosion behaviour of AZ31 magnesium alloy with different grain sizes in simulated biological fluids. Acta Biomater 2009; 6: 1763-1771.

17. Jiang B, Gao L, Huang G, et al. Effect of extrusion processing parameters on microstructure and mechanical properties of as-extruded AZ31 sheets. T Nonferr Metal Soc 2008; 18: 160-164.

18. Chen Y, Wang Q, Peng J, et al. Effects of extrusion ratio on the microstructure and mechanical properties of AZ31 Mg alloy. J Mater Process Tech 2007; 182: 281-285.

19. Shahzad M and Wagner L. Influence of extrusion parameters on microstructure and texture developments, and their effects on mechanical properties of the magnesium alloy AZ80. Mater Sci Eng 2009; 506: 141-147.

20. Witte $\mathbf{F}$, Fischer $\mathbf{J}$, Nellesen $\mathbf{J}$, et al. In vitro and in vivo corrosion measurements of magnesium alloys. Biomaterials 2006; 27: 1013-1018.

21. Krause A, Von der Höh N, Bormann D, et al. Degradation behaviour and mechanical properties of magnesium implants in rabbit tibiae. $J$ Mater Sci 2010; 45: 624-632.

22. Ullmann B, Reifenrath J, Dziuba D, et al. In vivo degradation behaviour of the magnesium alloy LANd442 in rabbit tibiae. Materials 2011; 4: 2197-2218.
23. Witte F, Fischer J, Nellesen J, et al. In vivo corrosion and corrosion protection of magnesium alloy LAE442. Acta Biomater 2010; 6: 1792-1799.

24. Makar $\mathrm{G}$ and Kruger J. Corrosion of magnesium. Int Mater Rev 1993; 38: 138-153.

25. Song G. Recent progress in corrosion and protection of magnesium alloys. Adv Eng Mater 2005; 7: 563-586.

26. Atrens A, Liu M and Zainal AN. Corrosion mechanism applicable to biodegradable magnesium implants. Mater Sci Eng B: Adv 2011; 176: 1609-1636.

27. Zhang E, Xu L, Yu G, et al. In vivo evaluation of biodegradable magnesium alloy bone implant in the first 6 months implantation. J Biomed Mater Res A 2009; 90: 882-893.

28. Li L, Gao J and Wang Y. Evaluation of cyto-toxicity and corrosion behavior of alkali-heat-treated magnesium in simulated body fluid. Surf Coat Technol 2004; 185: 92-98.

29. Song Y, Shan D, Chen R, et al. Biodegradable behaviors of AZ31 magnesium alloys in simulated body fluid. Mater Sci Eng C Mater Biol Appl 2009; 29: 1039-1045.

30. ASTM G31-72:2004. Standard practice for laboratory immersion corrosion testing of metals. American Society for Testing and Materials.

31. Dziuba D, Meyer-Lindenberg A, Seitz J-M, et al. Long term in vivo degradation behaviour and biocompatibility of the magnesium alloy ZEK100 for use as biodegradable bone implant. Acta Biomater. Epub ahead of print 23 August 2012. DOI: 10.1016/j.actbio.2012.08.028.

32. Lambotte A. L'utilisation du magnesium comme materiel perdu dans l'osteosynthèse. Bull Mém Soc Nat Chir 1932; 28: 1325-1334.

33. McBride ED. Absorbable metal in bone surgery. JAMA 1938; 111: 2464-2467.

34. Sugiyama T, Price JS and Lanyon LE. Functional adaptation to mechanical loading in both cortical and cancellous bone is controlled locally and is confined to the loaded bones. Bone 2010; 46: 314-321.

35. Lambers FM, Schulte FA, Kuhn G, et al. Mouse tail vertebrae adapt to cyclic mechanical loading by increasing bone formation rate and decreasing bone resorption rate as shown by time-lapsed in vivo imaging of dynamic bone morphometry. Bone 2011; 49: 1340-1350.

36. Witte F, Ulrich H, Palm C, et al. Biodegradable magnesium scaffolds: part II: peri-implant bone remodeling. J Biomed Mater Res A 2007; 81: 757-765.

37. Kubota K. Review processing and mechanical properties of fine-grained magnesium alloys. J Mater Sci 1999; 34: 2255-2262. 